\title{
NEW INTEGRALS INVOLVING BESSEL FUNCTIONS
}

BY

\author{
F. M. RAGAB
}

Ein Shams University, Abbassia, Cairo

The formula [T. M. MacRobert, Functions of a Complex Variable, $4^{\text {th }}$ ed., Glasgow 1954 , p. 406, (1)] namely

$$
K_{m}(x) K_{n}(x)=\frac{\sqrt{\pi}}{4 \pi x} \sum_{i,-i} \frac{1}{i} E\left(\frac{1+m+n}{2}, \frac{1+m-n}{2}, \frac{1+n-m}{2}, \frac{1-n-m}{2}: \frac{1}{2}: e^{i \pi} x^{2}\right),
$$

will be used to evaluate a large number of integrals involving Bessel Functions which are all entirely new.

Thus from (1), loc. cit., and formula (1)

$$
\int_{0}^{\infty} e^{-\lambda} \lambda^{k-1} E\left(p ; \alpha_{r}: q ; \varrho_{s}: \frac{x}{\lambda^{n}}\right) d \lambda=(2 \pi)^{1-\frac{1}{n} n} \cdot n^{k-\frac{1}{2}} E\left(p+n ; \alpha_{r}: q ; \varrho_{s}: \frac{x}{n^{n}}\right) \text {, }
$$

where

$$
R(k)>0, \alpha_{p+\nu+1}=\frac{k+\nu}{n}, \quad \nu=0,1,2, \ldots, n-1,
$$

one gets, if $x$ is real and positive,

$$
\begin{aligned}
& \int_{0}^{\infty} e^{-\lambda} \lambda^{k-1} K_{m}\left(\frac{x}{\lambda}\right) K_{n}\left(\frac{x}{\lambda}\right) d \lambda \\
& =\frac{2^{k-2}}{\pi x} \sum_{i,-i} \frac{1}{i} E\left(\frac{1+m+n}{2}, \frac{1+m-n}{2}, \frac{1+n-m}{2}, \frac{1-n-m}{2}, \frac{k+1}{2}, \frac{k+2}{2}: \frac{1}{2}: \frac{e^{i \pi} x^{2}}{4}\right) .
\end{aligned}
$$

Also from (1) and formula (2)

$$
\begin{aligned}
& \int_{0}^{\infty} e^{-\lambda} \lambda^{k-1} E\left(p ; \alpha_{r}: q ; \varrho_{s}: \lambda^{m} x\right) d \lambda=\pi \operatorname{cosec}(k \pi)(2 \pi)^{\frac{1}{m-1}} m^{k-\frac{1}{1}} \times \\
& \left.\times E\left(p ; \alpha_{r}: 1-\frac{k}{m}, 1-\frac{k+1}{m}, \ldots, 1-\frac{k+m-1}{m}, \varrho_{1}, \ldots, \varrho_{Q}: e^{ \pm m \pi i} m^{m} z\right)\right)+
\end{aligned}
$$

1-563801. Acta Mathematica. 95. Imprimé le 18 fóvrier 1956. 
2

F. M. RAGA

$$
\begin{aligned}
& +2^{\frac{1}{s-\frac{m}{2}} \pi^{t+\frac{m}{2}}} \sum_{v=0}^{m-1} \frac{(-1)^{\nu+1} m^{-1-v} z^{-(k+v) / m}}{\sin \left(\frac{k+v}{m} \pi\right) \prod_{s=1}^{p} \sin \frac{s \pi}{m} \prod_{t=1}^{m-v-1} \sin \frac{t \pi}{m}} \times \\
& \times E\left(\begin{array}{l}
p ; \alpha_{r}+(k+v) / m: e^{ \pm m i} m^{m} z \\
1+\frac{k+v}{m}, 1+\frac{1}{m}, \ldots, 1+\frac{v}{m}, 1-\frac{1}{m}, \ldots, 1-\frac{m-v-1}{m}, \varrho_{1}+\frac{k+v}{m}, \ldots, \varrho_{Q}+\frac{k+v}{m}
\end{array}\right),
\end{aligned}
$$

where $m$ is a positive integer (equals 2 in this case),

$$
R\left(m \alpha_{r}+k\right)>0, \quad r=1,2,3, \ldots, p,|\operatorname{amp} z|<\pi,
$$

one gets

$$
\begin{aligned}
& \int_{0}^{\infty} e^{-\lambda} \lambda^{k-1} K_{m}(x \lambda) K_{n}(x \lambda) d \lambda \\
& =\frac{\sqrt{\pi} \Gamma\left(\frac{m+n+k}{2}\right) \Gamma\left(\frac{m-n+k}{2}\right) \Gamma\left(\frac{n-m+k}{2}\right) \Gamma\left(\frac{-n-m+k}{2}\right)}{4 \Gamma\left(\frac{1+k}{2}\right) \Gamma\left(\frac{k}{2}\right)} x^{-k} \times \\
& \times{ }_{4} F_{3}\left(\frac{m+n+k}{2}, \frac{m-n+k}{2}, \frac{n-m+k}{2}, \frac{-n-n+k}{2} ; \frac{1}{2}, \frac{k}{2}, \frac{k+1}{2} ; \frac{1}{4 x^{2}}\right)- \\
& -\frac{\pi \Gamma\left(\frac{m+n+1+k}{2}\right) \Gamma\left(\frac{m-n+k+1}{2}\right) \Gamma\left(\frac{n-m+k+1}{2}\right) \Gamma\left(\frac{-n-m+k+1}{2}\right)}{8 \Gamma\left(\frac{3}{2}\right) \Gamma\left(\frac{k+1}{2}\right) \Gamma\left(\frac{k+2}{2}\right)} x^{-k-1} \times \\
& \quad \times F_{4}\left(\frac{1+m+n+k}{2}, \frac{1+m-n+k}{2}, \frac{1+n-m+k}{2}, \frac{1-n-m+k}{2} ; \frac{3}{2}, \frac{1+k}{2}, \frac{2+k}{2} ; \frac{1}{4 x^{2}}\right),
\end{aligned}
$$

where

$$
R(x)>0, R(k \pm m \pm n)>0 .
$$

In particular when $n=m$ the last formula becomes

$$
\begin{aligned}
& \int_{0}^{\infty} e^{-\lambda} \lambda^{k-1}\left\{K_{n}(x \lambda)\right\}^{2} d \lambda \\
& =\frac{\sqrt{\pi} \Gamma\left(n+\frac{k}{2}\right) \Gamma\left(-n+\frac{k}{2}\right) \Gamma\left(\frac{k}{2}\right)}{4 \Gamma\left(\frac{1+k}{2}\right)} x_{3}^{-k} F_{2}\left(\begin{array}{c}
\left.n+\frac{k}{2},-n+\frac{k}{2}, \frac{k}{2} ; \frac{1}{4 x^{2}}\right)- \\
\frac{1}{2}, \frac{1}{2}+\frac{1}{2} k
\end{array}\right) \\
& -\frac{\pi \Gamma\left(n+\frac{k+1}{2}\right) \Gamma\left(-n+\frac{k+1}{2}\right) \Gamma\left(\frac{k+1}{2}\right)}{8 \Gamma\left(\frac{3}{2}\right) \Gamma\left(1+\frac{1}{2} k\right)} x^{-k-1}{ }_{3} F_{2}\left(\begin{array}{c}
\left.n+\frac{k+1}{2},-n+\frac{k+1}{2}, \frac{k+1}{2} ; \frac{1}{4 x^{2}}\right) \\
\frac{3}{2}, 1+\frac{k}{2}
\end{array}\right), ~
\end{aligned}
$$


where

$$
R(k)>0, R(k \pm 2 n)>0, R(x)>0 .
$$

From (1) and formula (3)

$$
4 \int_{0}^{\infty} \lambda^{m-1} K_{n}(2 \lambda) E\left(p ; \alpha_{r}: q ; \varrho_{s}: \frac{x}{\lambda^{2}}\right) d \lambda=E\left(p+2 ; \alpha_{r}: q ; \varrho_{s}: x\right)
$$

where

$$
\alpha_{p+1}=\frac{m+n}{2}, \alpha_{p+2}=\frac{m-n}{2}, R(m \pm n)>0,
$$

one gets

$$
\begin{aligned}
& \int_{0}^{\infty} \lambda^{k-1} K_{l}(2 \lambda) K_{m}\left(\frac{x}{\lambda}\right) K_{n}\left(\frac{x}{\lambda}\right) d \lambda=\frac{1}{16 \sqrt{\pi} \cdot x} \times \\
& \quad \times \sum_{i,-i} \frac{1}{i} E\left(\frac{m+n+1}{2}, \frac{m-n+1}{2}, \frac{n-m+1}{2}, \frac{-n-m+1}{2}, \frac{k+l+1}{2}, \frac{k-l+1}{2}: \frac{1}{2}: e^{i \pi} x^{2}\right),
\end{aligned}
$$

where $R(x)>0$ and the restrictions necessary for (7) can be removed by analytical continuation.

When $n=m$, (8) gives, if $R(x)>0$,

$$
\begin{aligned}
\int_{0}^{\infty} \lambda^{k-1} K_{l}(2 \lambda)\left\{K_{n}\left(\frac{x}{\lambda}\right)\right\}^{2} d \lambda & \\
& =\frac{1}{16 \sqrt{\pi} \cdot x} \sum_{i,-i} \frac{1}{i} E\left(\frac{1}{2}+n, \frac{1}{2}-n, \frac{1}{2}, \frac{k+l+1}{2}, \frac{k-l+1}{2}:: e^{i \pi} x^{2}\right) .
\end{aligned}
$$

From (1) and formula (4)

$$
\begin{aligned}
4 i \pi \int_{0}^{\infty} \lambda^{m-1} J_{n}(2 \lambda) E & \left(p ; \alpha_{r}: q ; \varrho_{s}: \frac{z}{\lambda^{2}}\right) d \lambda \\
& =i^{m-n} E\left(p+2 ; \alpha_{r}: q ; \varrho_{s}: z e^{-i \pi}\right)-i^{n-m} E\left(p+2 ; \alpha_{r}: q ; \varrho_{s}: z e^{i \pi}\right)
\end{aligned}
$$

where

$$
R(m+n)>0, R\left(\frac{3}{2}-m+2 \alpha_{r}\right)>0, r=1,2,3, \ldots, p, \quad \alpha_{p+1}=\frac{m+n}{2}, \quad \alpha_{p+2}=\frac{m-n}{2}
$$

and $z$ is real and positive, one gets

$$
\int_{0}^{\infty} \lambda^{k-1} J_{l}(2 \lambda) K_{m}\left(\frac{x}{\lambda}\right) K_{n}\left(\frac{x}{\lambda}\right) d \lambda
$$




$$
\begin{aligned}
& =\frac{e^{\frac{i \pi}{2}(k-l-1)}}{16 \pi^{\frac{3}{2}} \cdot x}\left[\begin{array}{c}
E\left(\frac{1+m+n}{2}, \frac{1+m-n}{2}, \frac{1+n-m}{2}, \frac{1-n-m}{2}, \frac{1+k+l}{2}, \frac{1+k-l}{2}: \frac{1}{2}: x^{2}\right)- \\
-E\left(\frac{1+m+n}{2}, \frac{1+m-n}{2}, \frac{1+n-m}{2}, \frac{1-n-m}{2}, \frac{1+k+l}{2}, \frac{1+k-l}{2} ; \frac{1}{2}: e^{-2 i \pi} x^{2}\right)
\end{array}\right]- \\
& -\frac{e^{\frac{i \pi}{2}(l-k-3)}}{16 \pi^{\frac{3}{2}} \cdot x}\left[\begin{array}{c}
E\left(\frac{1+m+n}{2}, \frac{1+m-n}{2}, \frac{1+n-m}{2}, \frac{1-n-m}{2}, \frac{1+k+l}{2}, \frac{1+k-l}{2}: \frac{1}{2}: e^{2 i \pi} x^{2}\right)- \\
-E\left(\frac{1+m+n}{2}, \frac{1+m-n}{2}, \frac{1+n-m}{2}, \frac{1-n-m}{2}, \frac{1+k+l}{2}, \frac{1+k-l}{2}: \frac{1}{2}: x^{2}\right)
\end{array}\right] \\
& \text { where } \\
& R(x)>0, R\left(\frac{3}{2}-k \pm m \pm n\right)>0 .
\end{aligned}
$$

When $n=m$, the last formula becomes

$$
\begin{aligned}
& \int_{0}^{\infty} \lambda^{k-1} J_{l}(2 \lambda)\left\{K_{n}\left(\frac{x}{\lambda}\right)\right\}^{2} d \lambda
\end{aligned}
$$

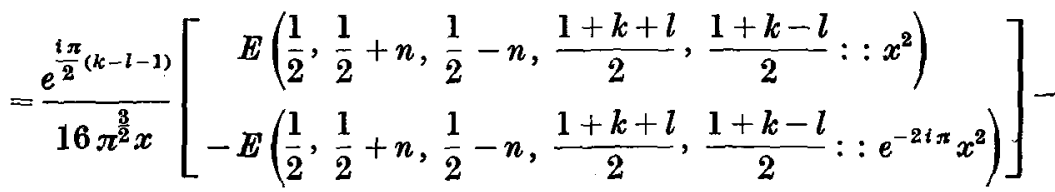

$$
\begin{aligned}
& -\frac{e^{\frac{i n}{2}(l-k-3)}}{16 \pi^{\frac{3}{2}} \cdot x}\left[\begin{array}{r}
E\left(\frac{1}{2}, \frac{1}{2}+n, \frac{1}{2}-n, \frac{1+k+l}{2}, \frac{1+k-l}{2}:: e^{2 i \pi} x^{2}\right) \\
-E\left(\frac{1}{2}, \frac{1}{2}+n, \frac{1}{2}-n, \frac{1+k+l}{2}, \frac{1+k-l}{2}:: x^{2}\right)
\end{array}\right], \\
& R(x)>0, R\left(\frac{3}{2}-k\right)>0, R\left(\frac{3}{2}-k \pm 2 n\right)>0 .
\end{aligned}
$$

From (1) and formula (5)

$$
\begin{aligned}
& \int_{0}^{\infty} \lambda^{k-1} K_{n}(\lambda) E\left(p ; \alpha_{r}: q ; \varrho_{s}: z \lambda^{2}\right) d \lambda \\
& =2^{k-2} \frac{\pi^{2}}{\sin \left(\frac{k+n}{2} \pi\right) \sin \left(\frac{k-n}{2} \pi\right)} E\left(p ; \alpha_{r}: 1-\frac{k+n}{2}, 1-\frac{k-n}{2}, \varrho_{1}, \ldots, \varrho_{a}: 4 z\right) \\
& +\sum_{n,-n} \frac{\pi^{2} 2^{-n-2}}{\sin \left(\frac{k+n}{2} \pi\right) \sin (n \pi)} z^{-\left(\frac{k+n}{2}\right)} E\left(\begin{array}{l}
\alpha_{1}+\frac{k+n}{2}, \ldots, \alpha_{p}+\frac{k+n}{2}: 4 z \\
\left.1+\frac{k+n}{2}, 1+n, \varrho_{1}+\frac{k+n}{2}, \ldots, \varrho_{a}+\frac{k+n}{2}\right)
\end{array}\right)
\end{aligned}
$$

where $p \geq q+1, R\left(k \pm n+2 \alpha_{r}\right) \geq 0, r=1,2,3, \ldots, p, \mid$ amp $z \mid<\pi$, one gets 
$\int_{0}^{\infty} \lambda^{k-1} K_{l}(\lambda) K_{m}(x \lambda) K_{n}(x \lambda) d \lambda$

$=-\sum_{l,-l}\left[\begin{array}{l}\frac{2^{-l-3} \pi^{\frac{3}{2}} \Gamma\left(\frac{k+l+m+n}{2}\right) \Gamma\left(\frac{k+l+m-n}{2}\right) \Gamma\left(\frac{k+l+n-m}{2}\right) \Gamma\left(\frac{k+l-m-n}{2}\right)}{\sin (l \pi) \Gamma\left(\frac{1+k+l}{2}\right) \Gamma\left(\frac{k+l}{2}\right) \Gamma(1+l) \cdot x} \\ \times{ }_{4} F_{3}\left(\begin{array}{l}\left.\frac{k+l+m+n}{2}, \frac{k+l+m-n}{2}, \frac{k+l+n-m}{2}, \frac{k+l-n-m}{2} ; \frac{1}{4 x^{2}}\right) \\ \frac{1+k+l}{2}, \frac{k+l}{2}, 1+l\end{array}\right)\end{array}\right]$,

where

$$
R(x)>0, R(k \pm l \pm m \pm n)>0 .
$$

In particular when $n=m_{1}$ the last formula gives

$$
\begin{aligned}
\int_{0}^{\infty} \lambda^{k-1} K_{l}(\lambda)\left\{K_{n}(x \lambda)\right\}^{2} d \lambda \\
=-\sum_{l_{1}-l}\left[\begin{array}{l}
\frac{2^{-l-3} \pi^{\frac{3}{2}} \Gamma\left(\frac{1}{2} k+\frac{1}{2} l+n\right) \Gamma\left(\frac{1}{2} k+\frac{1}{2} l-n\right) \Gamma\left(\frac{1}{2} k+\frac{1}{2} l\right)}{\sin (l \pi) \Gamma\left(\frac{1+k+l}{2}\right) \cdot x \cdot \Gamma(1+l)} x^{-(k+l-1)} \\
\times{ }_{3} F_{2}\left(\frac{1}{2} k+\frac{1}{2} l+n, \frac{1}{2} k+\frac{1}{2} l-n, \frac{k+l}{2} ; \frac{1+k+l}{2}, 1+l ; \frac{1}{4 x^{2}}\right)
\end{array}\right],
\end{aligned}
$$

where

$$
R(x)>0, R(k \pm l \pm 2 n)>0, R(k \pm l)>0 .
$$

From (1) and formula (6)

$$
\begin{aligned}
& \int_{0}^{\infty} e^{-\mu} I_{n}(\mu) \mu^{m-1} E\left(p ; \alpha_{r} ; q ; \varrho_{s}: z / \mu^{2}\right) d \mu \\
& =\frac{1}{2 \sqrt{2}} \frac{\sin \pi(m-n)}{\cos (\pi m)} E\left\{\begin{array}{l}
\alpha_{1}, \ldots, \alpha_{p}, \frac{m+n}{2}, \frac{m-n}{2}, \frac{m-n+1}{2}, \frac{m+n+1}{2}: z \\
\frac{3}{4}+\frac{1}{2} m, \frac{1}{4}+\frac{1}{2} m, \varrho_{1}, \ldots, \varrho_{q}
\end{array}\right\}- \\
& -\frac{1}{4 \sqrt{2} \cdot \pi} \frac{\cos (n \pi)}{\sin \pi\left(\frac{m}{2}-\frac{1}{4}\right)} z^{\frac{m}{2}-\frac{1}{4}} E\left\{\begin{array}{l}
\alpha_{1}+\frac{1}{4}-\frac{m}{2}, \ldots, \alpha_{p}+\frac{1}{4}-\frac{m}{2}, \frac{1}{4}+\frac{n}{2}, \frac{3}{4}+\frac{n}{2}, \frac{1}{4}-\frac{n}{2}, \frac{3}{4}-\frac{n}{2}: z \\
\frac{5}{4}-\frac{m}{2}, \frac{1}{2}, \varrho_{1}+\frac{1}{4}-\frac{m}{2}, \ldots, \varrho_{a}+\frac{1}{4}-\frac{m}{2}
\end{array}\right\}- \\
& -\frac{1}{4 \sqrt{2} \cdot \pi} \frac{\cos (n \pi)}{\sin \pi\left(\frac{3}{4}-\frac{m}{2}\right)} z^{\frac{m}{2}-\frac{3}{4}} E\left\{\begin{array}{l}
\alpha_{1}+\frac{3}{4}-\frac{m}{2}, \ldots, \alpha_{p}+\frac{3}{4}-\frac{m}{2}, \frac{3}{4}+\frac{n}{2}, \frac{5}{4}+\frac{n}{2}, \frac{3}{4}-\frac{n}{2}, \frac{5}{4}-\frac{n}{2}: z \\
\frac{7}{4}-\frac{m}{2}, \frac{3}{2}, \varrho_{1}+\frac{3}{4}-\frac{m}{2}, \ldots, \varrho_{a}+\frac{3}{4}-\frac{m}{2}
\end{array}\right\},(1,)
\end{aligned}
$$


where

$$
R(n+m)>0, R\left(2 \alpha_{r}-m+\frac{1}{2}\right)>0, r=1,2,3, \ldots, p,|\operatorname{amp} z|<\pi,
$$

one gets the following formula

$$
\begin{aligned}
& \int_{0}^{\infty} e^{-\lambda} \lambda^{k-1} I_{l}(\lambda) K_{m}\left(\frac{x}{\lambda}\right) K_{n}\left(\frac{x}{\lambda}\right) d \lambda=\frac{1}{4 x \sqrt{\pi}} \times
\end{aligned}
$$

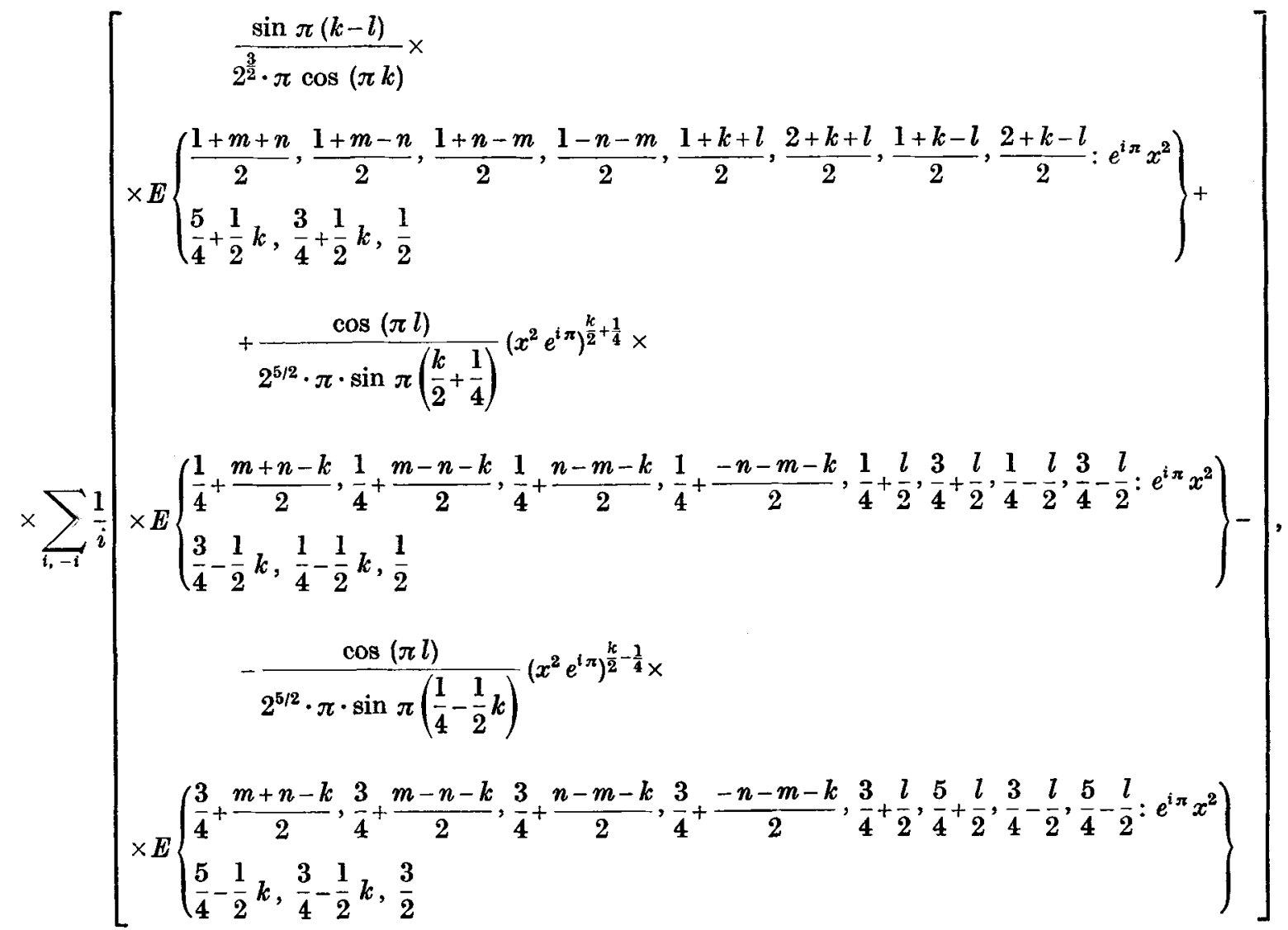

where

$$
R(x)>0, R\left(\frac{1}{2}-k \pm m \pm n\right)>0
$$

When $n=m$, the last formula becomes 


$$
\begin{aligned}
& \int_{0}^{\infty} e^{-\lambda} \lambda^{k-1} I_{l}(\lambda)\left\{K_{n}\left(\frac{x}{\lambda}\right)\right\}^{2} d \lambda=\frac{1}{4 \sqrt{\pi} \cdot x} \times
\end{aligned}
$$

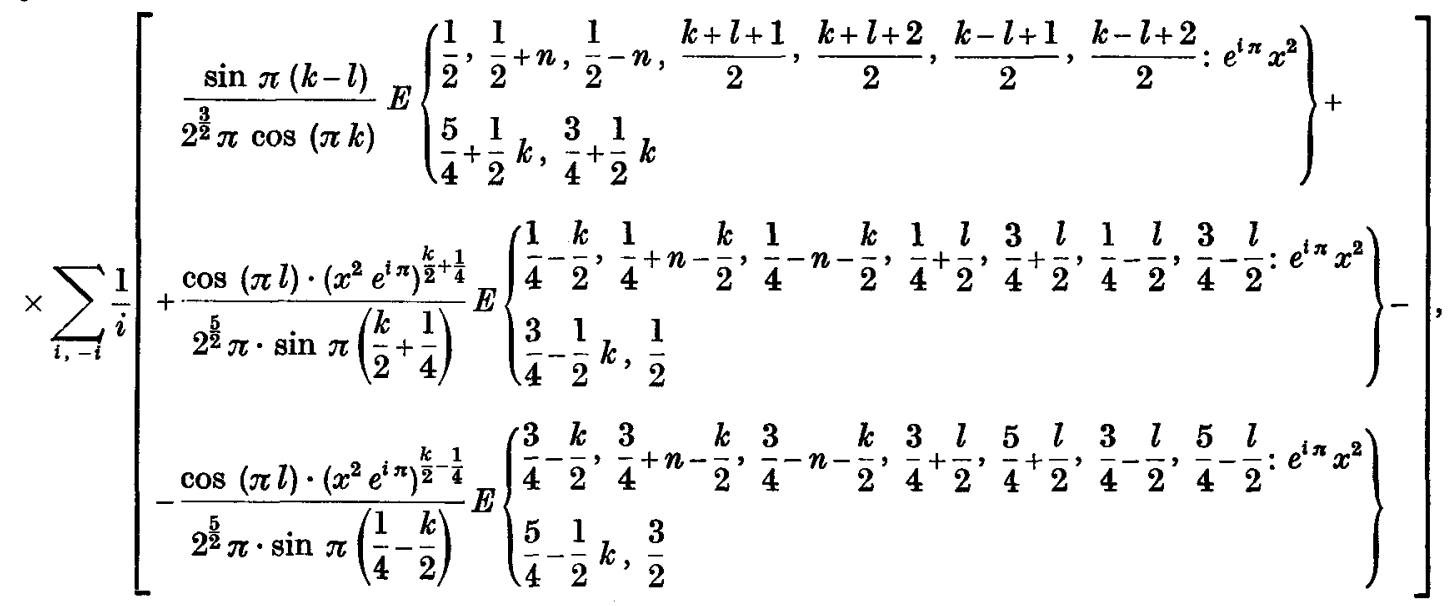

where

$$
R(x)>0, R\left(\frac{1}{2}-k\right)>0, R\left(\frac{1}{2}-k \pm 2 n\right)>0 .
$$

Finally from (1) and formula (7) $\int_{0}^{\infty} e^{-\mu} \mathrm{I}_{n}(\mu) \mu^{m-1} E\left(p ; \alpha_{r}: q ; \varrho_{s}: z \mu^{2}\right) d \mu$ $=\frac{\pi}{\sqrt{2} \sin \pi(m+n)} E\left\{\begin{array}{l}\alpha_{1}, \ldots, \alpha_{r}, \frac{1}{4}-\frac{m}{2}, \frac{3}{4}-\frac{m}{2}: z \\ 1-\frac{m+n}{2}, \frac{1}{2}-\frac{m+n}{2}, \varrho_{1}, \ldots, \varrho_{q}, 1+\frac{n-m}{2}, \frac{1}{2}+\frac{n-m}{2}\end{array}\right\}-$ $-\frac{\pi \cdot z^{-\left(\frac{m+n}{2}\right)}}{2 \sqrt{2} \sin \pi\left(\frac{m+n}{2}\right)} E\left\{\begin{array}{l}\alpha_{1}+\frac{m+n}{2}, \ldots, \alpha_{p}+\frac{m+n}{2}, \frac{1}{4}+\frac{n}{2}, \frac{3}{4}+\frac{n}{2}: z \\ 1+\frac{m+n}{2}, \frac{1}{2}, \varrho_{1}+\frac{m+n}{2}, \ldots, \varrho_{q}+\frac{m+n}{2}, 1+n, \frac{1}{2}+n\end{array}\right\}-$ $-\frac{\pi \cdot z^{-\left(\frac{m+n+1}{2}\right)}}{2 \sqrt{2} \cos \pi\left(\frac{m+n}{2}\right)} E\left\{\begin{array}{l}\alpha_{1}+\frac{m+n+1}{2}, \ldots, \alpha_{p}+\frac{m+n+1}{2}, \frac{3}{4}+\frac{n}{2}, \frac{5}{4}+\frac{n}{2}: z \\ 1+\frac{m+n+1}{2}, \frac{3}{2}, \varrho_{1}+\frac{m+n+1}{2}, \ldots, \varrho_{q}+\frac{m+n+1}{2}, \frac{3}{2}+n, 1+n\end{array}\right\}$

where

one gets

$$
R\left(n+m+2 \alpha_{r}\right)>0, r=1,2,3, \ldots, p, R\left(\frac{1}{2}-m\right)>0,|\operatorname{amp} z|<\pi
$$




$$
\begin{aligned}
& \int_{0}^{\infty} e^{-\lambda} I_{l}(\lambda) K_{m}(x \lambda) K_{n}(x \lambda) d \lambda \\
& =\frac{V_{\pi} \Gamma\left(\frac{k+l+m+n}{2}\right) \Gamma\left(\frac{k+l+m-n}{2}\right) \Gamma\left(\frac{k+l+n-m}{2}\right) \Gamma\left(\frac{k+l-m-n}{2}\right) \Gamma\left(\frac{1}{4}+\frac{l}{2}\right) \Gamma\left(\frac{3}{4}+\frac{l}{2}\right)}{\left.2^{\frac{5}{2}} \Gamma\left(\frac{1}{2}+\frac{1}{2} k+\frac{1}{2} l\right) \Gamma\left(\frac{1}{2}\right) \Gamma\left(\frac{1}{2} k+\frac{1}{2} l\right) \Gamma(1+l)\right) \Gamma\left(\frac{1}{2}+l\right) \cdot x} x^{-(k+l-1)} \times \\
& \times_{6} F_{5}\left(\begin{array}{l}
\frac{k+l+m+n}{2}, \frac{k+l+m-n}{2}, \frac{k+l+n-m}{2}, \frac{k+l-n-m}{2}, \frac{1}{4}+\frac{l}{2}, \frac{3}{4}+\frac{l}{2}, \frac{1}{x^{2}} \\
\frac{k+l+1}{2}, \frac{k+l}{2}, \frac{1}{2}, 1+l, \frac{1}{2}+l
\end{array}\right) \\
& -\frac{\sqrt{\pi} \Gamma\left(\frac{k+l+m+n+1}{2}\right) \Gamma\left(\frac{k+l+m-n+1}{2}\right) \Gamma\left(\frac{k+l+n-m+1}{2}\right) \Gamma\left(\frac{k+l-n-m+1}{2}\right) \Gamma\left(\frac{3}{4}+\frac{l}{2}\right) \Gamma\left(\frac{5}{4}+\frac{l}{2}\right)}{2^{\frac{5}{2}} \Gamma\left(1+\frac{k+l}{2}\right) \Gamma\left(\frac{3}{2}\right) \Gamma\left(\frac{1+k+l}{2}\right) \Gamma(1+l) \Gamma\left(\frac{3}{2}+l\right) \cdot x} x^{-(k+1) \times} \\
& \times_{8} F_{5}\left(\begin{array}{l}
\frac{k+l+m+n+1}{2}, \frac{k+l+m-n+1}{2}, \frac{k+l+n-m+1}{2}, \frac{k+l-n-m+1}{2}, \frac{3}{4}+\frac{l}{2}, \frac{5}{4}+\frac{l}{2} ; \frac{1}{x^{2}} \\
\frac{2+k+l}{2}, \frac{3}{2}, \frac{1+k+l}{2}, 1+l, \frac{3}{2}+l
\end{array}\right) \text {, } \\
& R(x)>0, R(k+l \pm m \pm n)>0 .
\end{aligned}
$$

Addendum.-It may be noted that the last formulae may serve as a basis for discussion of the asymptotic behaviour of the integrals for large values of $|z|$ since some of the above integrals were evaluated in terms of MacRobert's $E$ function whose asymptotic expansion was given by him (C. V., p. 358) ${ }^{1}$ and the rest were evaluated in terms of ordinary generalized hypergeometric function whose asymptotic expansion has been investigated by several writers [E. W. Barnes, Proc. Lond. Math. Soc., (2) 5 (1906), 249-297; E. M. Wright, Journ. Lond. Math. Soc., 10 (1935), 286-295; and C. S. Meyer, Proc. K. Akad. v. Wetenschappen, Amsterdam, XLIX (1946), 1165-1175)].

\section{References}

[1]. T. M. MacRobert, Proc. Glasgow Math. Assoc. I, 191 (1953), p. 191, form. (9).

[2]. F. M. RAgAB, Proc. Glasgow Math. Assoc. II (1954), p. 77, form. (1).

[3]. T. M. MacRobert, Phil. Mag., Ser. 7, XXXI (1941), p. 258.

[4]. F. M. RAGAB, Proc. Glasgow Math. Assoc. I (1951), p. 8, form. (6).

[5]. —, Proc. Glasgow Math. Assoc. II (1953), p. 52, form. (2).

[6]. - - Proc. Glasgow Math. Assoc. II (1954), p. 82, form. (7).

[7]. -, Proc. Glasgow Math. Assoc. II (1954), p. 87, form. (8).

$1 \mathrm{C}$. V. denotes the book by MacRobert referred to in the beginning of this paper. 